\title{
Nash equilibria of a generic networking game with applications to circuit-switched networks
}

\author{
Youngmi Jin and George Kesidis \\ Electrical Engineering and Computer Science and Engineering Departments \\ Pennsylvania State University, University Park PA 16803 \\ Email: yzj100@psu.edu, kesidis@engr.psu.edu
}

\begin{abstract}
A generic mechanism for end-user transmission rate control into a differentiated services Internet is formulated and basic results of corresponding Nash equilibria are proved. We consider specific examples of the mechanism including additive increase and multiplicative decrease inspired by present day TCP congestion control. For the example of users sharing access to a bandwidth resource via resizable provisioned label-switched paths (MPLS), we study the equilibria and the performance of the generic mechanism and give analytical results on convergence to equilibria. The fairness of the resulting equilibria when user demands exceed available network resources is also studied.
\end{abstract}

\section{INTRODUCTION}

Research into Internet flow control involving pricing is receiving more attention as mechanisms for differential qualitiesof-service (QoSs) are being deployed. Imposing usage-based charges encourages users to behave in more reasonable ways, e.g., by discouraging the use of higher-priority (cheaper) service classes for "best-effort" applications with flexible QoS requirements. Premium services include assured service (AS) and expedited forwarding (EF) under the differentiated services (diffserv) paradigm [2], [19] and provisioned labelswitched paths (LSPs) under the multiprotocol label switching (MPLS) paradigm [5], [4]. Preliminary studies of issues of pricing and user access mechanisms for differentiated services are given in [21], [17]. In [18], a pricing scheme is considered wherein network traffic is classified into different priorities, and users choose the priorities and are charged by the network according to the chosen priority. The dynamics are modeled as a non-cooperative game and the existence of an equilibrium for the game model and the resource allocation among the users are studied. Recently, in [13], we devised a two-level user access mechanism in which users select a class-of-service $(\mathrm{CoS})$ based on their ability to pay and on the QoS need of the application under consideration by the user; the network links determine the "clearing" price and clearing CoS based on the mean backlog of a queue with a differential enqueue mechanism (as currently in practice); and the end-to-end clearing prices and CoSs are communicated to the user via a minimum-cost routing algorithm.

In [10], [11], a distributed constrained optimization problem to find flow-rates (bandwidth) which maximize the sum of

This research is supported by NSF ITR 0219747. each user's utility is studied. They showed that the optimization problem can be decomposed into two sub-problems for users and the network in which each user pursues his/her own net benefit (utility - cost) and the network finds optimum flow-rates given only each user's willingness to pay. Based on the decomposition, [11] proposes a decentralized algorithm based on duality wherein greedy users rapidly adjust their willingnesses to pay according to their previous set of network link prices and the network slowly adjusts user flow-rate grants and link prices according to a kind of proportional fairness rule. With the same "social welfare" objective, i.e., to find flow rates which maximize the sum of user utilities, a related decentralized dual approach is proposed in [16] in which users rapidly select transmission rates that maximize their own benefits and the network slowly adjusts link bandwidth prices in response.

This paper considers user dynamics in a circuit-switched Internet offering bandwidth-provisioned LSPs billed according to usage. We first suggest a generic mechanism for end-user transmission rate control (or, more generally, access control) to an Internet offering differentiated CoSs, which leads to a Nash equilibrium. Under this mechanism, users modify their access control parameter based on the quality-of-service they currently receive. Convergence to Nash equilibria [1] is studied under the assumption of synchronous user updates of their access control parameters. The results obtained herein can be extended to asynchronous (“decentralized") updates using techniques and assumptions described in [15].

A special case of our generic user access mechanism is TCP's slow-start congestion control policy (additive rate increase and multiplicative decrease) extended in a natural way to include differential and targeted user demands [13]. Our previous work on ALOHA [9] is another application of this generic mechanism.

This paper does not consider the dynamics between the users and network as the network adjusts prices in response to perceived user demand. We only study the user behavior for fixed network prices, specifically convergence to the Nash equilibrium (i.e., the "fast" dynamics in the dual algorithms of [10], [16]). Specifically, we specify potential functions that govern these dynamics.

This paper is organized as follows. In Section II, we give an overview of the generic game theoretic framework under 
consideration. In Sections III and IV, we study convergence to Nash equilibrium points of users for a fixed charge per circuit per unit time imposed by the network; in addition, we numerically explore the fairness of specific mechanisms when total user demand exceeds available network capacity. We conclude with a summary in Section V.

\section{A GENERIC FIXED POINT ITERATION LEADING TO NASH EQUILIBRIA}

In this section, we study the existence of equilibrium behavior of a population of $N$ users competing for resource units (bandwidth) which are offered for sale by a network. We formulate a generic noncooperative game that can be applied to several networking situations. Each user $n$ has an access control (or "input") parameter $\lambda_{n}$ and receives a certain amount $\theta_{n}(\underline{\lambda})$ of the network's resources where $\lambda \equiv\left(\lambda_{1}, \lambda_{2}, \ldots, \lambda_{N}\right)$, i.e., what the $n^{\text {th }}$ user receives may depend on the action taken by other users. We assume that for each $n$ :

- $\lambda_{n} \in\left[0, \lambda_{n}^{\max }\right]$ for some fixed $\lambda_{n}^{\max }>0$.

- $\theta_{n}(\underline{\lambda})$ is a nondecreasing function of $\lambda_{n}$.

- $\theta_{n}(\cdot)$ is continuous in $\prod_{n=1}^{N}\left[0, \lambda_{n}^{\max }\right]$ and differentiable with respect to $\lambda_{n}$.

- $\theta_{n}(\underline{\lambda})=0$ for all $\underline{\lambda}$ such that $\lambda_{n}=0$.

Alternatively, $\theta_{n}(\underline{\lambda})$ may be interpreted as the quality-ofservice (QoS) received by the $n^{\text {th }}$ user. The network charges a fixed amount $M$ per unit resource consumed.

Each user $n$ seeks to maximize his/her net utility, $U_{n}(\theta)-$ $M \theta$, where, for all $n$ :

- $U_{n}$ is nondecreasing and $U_{n}(0)=0$.

- $U_{n}^{\prime}$ is nonincreasing, i.e., $U_{n}$ is concave.

Thus, the maximum of the $n^{\text {th }}$ user's net benefit under usagebased costs is

$$
\arg \max _{\theta} U_{n}(\theta)-M \theta=\left(U_{n}^{\prime}\right)^{-1}(M) .
$$

We will assume that $\left(U_{n}^{\prime}\right)^{-1}(M)>0$. Note that if $\left(U_{n}^{\prime}\right)^{-1} \leq 0$ then the $n^{\text {th }}$ user will simply not use the network. The users sequentially adjust their control parameters $\underline{\lambda}$ in an attempt to make their received $\operatorname{QoS} \theta_{n}(\underline{\lambda})$ equal to their desired QoS $y_{n} \equiv\left(U_{n}^{\prime}\right)^{-1}(M)$. After the $j^{\text {th }}$ iteration/step, the user input parameter is, for each $n$ :

$$
\lambda_{n}^{j+1}=\min \left\{G\left(y_{n}, \theta_{n}\left(\underline{\lambda}^{j}\right), \lambda_{n}^{j}\right), \lambda_{n}^{\max }\right\} .
$$

where the jointly continuous function $G$ satisfies the following properties:

$$
G(y, \theta, \lambda) \begin{cases}\equiv \lambda & \text { if } y=\theta \\ >\lambda & \text { if } y>\theta \\ <\lambda & \text { if } y<\theta\end{cases}
$$

\section{A. Nash equilibria}

A fixed or equilibrium point of this iteration is any $\underline{\lambda}^{*} \in$ $\prod_{n=1}^{N}\left[0, \lambda_{n}^{\max }\right]$ such that

$$
\lambda_{n}^{*}=\min \left\{G\left(y_{n}, \theta_{n}\left(\underline{\lambda}^{*}\right), \lambda_{n}^{*}\right), \lambda_{n}^{\max }\right\} .
$$

By Brouwer's fixed point theorem [20], [3], there exists at least one such fixed point. Note that we are implicitly assuming that every user does not divulge his/her utility function and, in particular, does not divulge their "demand" $y_{n}$ for a given price $M$. Note that this fixed point iteration requires only that the users ascertain (estimate) their own received QoS, $\theta_{n}$, at each step $j$.

Lemma 1: (a) If $\lambda_{n}^{*}<\lambda_{n}^{\max }, \theta_{n}\left(\underline{\lambda}^{*}\right)=y_{n}$.

(b) $\theta_{n}\left(\underline{\lambda}^{*}\right) \leq y_{n}$.

Proof: From (3), $\lambda_{n}^{*}$ should be either $\lambda_{n}^{\max }$ or $G\left(y_{n}, \theta_{n}\left(\underline{\lambda}^{*}\right), \lambda_{n}^{*}\right)$ which is smaller than $\lambda_{n}^{\max }$. If $\lambda_{n}^{*}=$ $G\left(y_{n}, \theta_{n}\left(\underline{\lambda}^{*}\right), \lambda_{n}^{*}\right)<\lambda_{n}^{\max }$, (a) comes from the definition of $G$. If $\lambda_{n}^{*}=\lambda_{n}^{\max }$, then $G\left(y_{n}, \theta_{n}\left(\underline{\lambda}^{*}\right), \lambda_{n}^{*}\right) \geq \lambda_{n}^{\max }$. Hence, $\theta_{n}\left(\underline{\lambda}^{*}\right) \leq y_{n}$ holds.

Theorem 1: Each such fixed point is a Nash equilibrium point (NEP).

Proof: An immediate consequence of the previous lemma, the continuity assumption of each $\theta_{n}$ and the definition of $y_{n}$ is that all fixed points in the interior of $\prod_{n=1}^{N}\left[0, \lambda_{n}^{\max }\right]$ are NEPs, i.e.,

$$
\lambda_{n}^{*}=\arg \max _{0 \leq \lambda \leq \lambda_{n}^{\max }} U_{n}\left(\theta\left(\lambda ; \underline{\lambda}_{-n}^{*}\right)\right)-M \theta\left(\lambda ; \underline{\lambda}_{-n}^{*}\right)
$$

where $\left(\lambda ; \underline{\lambda}_{-n}^{*}\right)$ is the $N$-vector whose $k^{\text {th }}$ component is $\lambda_{k}^{*}$ for all $k \neq n$ and whose $n^{\text {th }}$ component is $\lambda$.

By the previous lemma, to show that a fixed point $\lambda^{*}$ on the boundary of $\prod_{n=1}^{N}\left[0, \lambda_{n}^{\max }\right]$, some of whose components are $\lambda_{n}^{\max }$ for some $n$ 's, is a NEP, we need to show that if

$$
\lambda_{n}^{*}=\lambda_{n}^{\max }
$$

then

$$
\begin{aligned}
\lambda_{n}^{\max } & =\arg \max _{0 \leq \lambda \leq \lambda_{n}^{\max }} f(\lambda) \quad \text { where } \\
f(\lambda) & =U_{n}\left(\theta_{n}\left(\lambda ; \underline{\lambda}_{-n}^{*}\right)\right)-M \theta_{n}\left(\lambda ; \underline{\lambda}_{-n}^{*}\right)
\end{aligned}
$$

and $\underline{\lambda}_{-n}^{*}=\left(\lambda_{1}^{*}, \ldots, \lambda_{n-1}^{*}, \lambda_{n+1}^{*}, \ldots, \lambda_{N}^{*}\right)$. This statement clearly follows if $f^{\prime} \geq 0$ ( $f$ is nondecreasing) over the whole interval $\left[0, \lambda_{n}^{\max }\right]$. By direct differentiation,

$$
\left.f^{\prime}(\lambda)=\left(U_{n}^{\prime}\left(\theta_{n}\left(\lambda ; \underline{\lambda}_{-n}^{*}\right)\right)-M\right)\right) \frac{\partial \theta_{n}\left(\lambda ; \underline{\lambda}_{-n}^{*}\right)}{\partial \lambda_{n}}
$$

First note that $\partial \theta_{n}\left(\lambda ; \underline{\lambda}_{-n}^{*}\right) / \partial \lambda_{n} \geq 0$ by assumption. By statement (b) of the previous lemma, $\theta_{n}\left(\underline{\lambda}^{*}\right) \leq y_{n} \equiv\left(U_{n}^{\prime}\right)^{-1}(M)$; therefore, because $U_{n}^{\prime}$ is nonincreasing by assumption,

$$
U_{n}^{\prime}\left(\theta_{n}\left(\underline{(}^{*}\right)\right)=U_{n}^{\prime}\left(\theta_{n}\left(\lambda_{n}^{\max } ; \underline{\lambda}_{-n}^{*}\right)\right) \geq M .
$$

Again, because $U_{n}^{\prime}$ is nonincreasing and because $\theta_{n}$ is nondecreasing in $\lambda_{n}$ (also by assumption), the previous inequality implies

$$
U_{n}^{\prime}\left(\theta_{n}\left(\lambda ; \underline{\lambda}_{-n}^{*}\right)\right) \geq M
$$


for all $\lambda \leq \lambda_{n}^{\max }$ as desired.

Definition 1: A NEP $\underline{\lambda}^{*}$ is Pareto optimal if, for any other NEP $\alpha^{*}$,

$$
U_{n}\left(\theta_{n}\left(\underline{\lambda}^{*}\right)\right)-M \theta_{n}\left(\underline{\lambda}^{*}\right) \geq U_{n}\left(\theta_{n}\left(\underline{\alpha}^{*}\right)\right)-M \theta_{n}\left(\underline{\alpha}^{*}\right)
$$

for all $n$.

An immediate consequence of the previous theorem and lemma is:

Theorem 2: Fixed points (NEPs) in the interior of $\prod_{n=1}^{N}\left[0, \lambda_{n}^{\max }\right]$ are Pareto optimal.

As we shall see, boundary NEPs are reached typically when total user demand exceeds network capacity, i.e., congestion. Note that Theorem 2 states that, at a boundary NEP, no user is receiving more than their demand which is a basic notion of fairness. A more specific criterion is that, at a boundary point $\underline{\lambda}^{*}, \theta_{n}\left(\underline{\lambda}^{*}\right) / y_{n}$ is a constant function of $n$, i.e., demand proportional fairness (which is different from proportional fairness of [10], [11]). Demand proportional fairness seems to be the natural extension of the "equal share" fairness achieved by TCP's slow start for a highly ideal model of the present-day Internet [6], [14].

\section{B. Specific user access mechanisms}

In this section, we will describe two specific user access mechanisms. The iterations (1) are additive increase and multiplicative decrease (AIMD) if

$$
G(y, \theta, \lambda)=\lambda-\alpha \lambda \mathbf{1}\{y<\theta\}+\delta \mathbf{1}\{y>\theta\}
$$

where $1-\alpha \in(0,1)$ is the multiplicative decrease factor (exceeded demand) and $\delta>0$ is the additive increase factor (demand not met).

To mitigate oscillations about an equilibrium point $\underline{\lambda}^{*}$ (even for asynchronous user updates), it is desirable to let the quantities $\alpha \rightarrow 1$ and $\delta \rightarrow 0$ (i.e., reduce the "step size") as $\theta \rightarrow y$. We therefore consider a modification of (5):

$$
\begin{aligned}
& G(y, \theta, \lambda)=\left(1-\alpha\left(1-\frac{y}{\theta}\right)\right) \lambda \mathbf{1}\{y<\theta\} \\
& +\left(\lambda+\delta\left(\frac{y}{\theta}-1\right)\right) \mathbf{1}\{y>\theta\}+\lambda \mathbf{1}\{y=\theta\}
\end{aligned}
$$

In the following, we study the convergence of (1) to NEPs for the multiplicative increase and multiplicative decrease (MIMD) policy, simply:

$$
G(y, \theta, \lambda)=\frac{y}{\theta} \lambda .
$$

We were unable to establish whether the fixed point iterations considered below are contraction mappings. As a result, we appealed to the Lyapunov theory of system stability and, to do so, considered the Jacobi update scheme. In any case, this technique provides more information about the nature of the NEPs than just uniqueness.

\section{Unconstrained Jacobi iteration}

Consider the following modification of the game (1) without the constraints $\lambda_{n}^{\max }$ :

$$
\underline{\lambda}^{j+1}=\underline{\lambda}^{j}+\varepsilon\left(F\left(\underline{\lambda}^{j}\right)-\underline{\lambda}^{j}\right)
$$

for a fixed small $\varepsilon>0$ where the $n^{\text {th }}$ component of the function $F:[0, \infty)^{N} \rightarrow[0, \infty)^{N}$ is

$$
F_{n}(\underline{\lambda})=G\left(y_{n}, \theta_{n}(\underline{\lambda}), \lambda_{n}\right) .
$$

This is simply the Jacobi update scheme, see equation (15) of [15]. For small $\varepsilon$, we can approximate (8) by the continuoustime game

$$
\dot{\lambda}(t)=F(\underline{\lambda}(t))-\underline{\lambda}(t) .
$$

The Jacobi iteration will be used to show the convergence of the fixed point iteration. In general, it is hard to show the fixed point iteration converges to the fixed point due to the nonlinearity of $\theta_{n}$ in the problems we will discuss with. To show the convergence to the interior fixed point, we will find a potential or Lyapunov function of (9).

\section{A NONCOOPERATIVE GAME FOR A CIRCUIT-SWITCHED SYSTEM}

Consider a group of $K$ circuits connecting an origin node to a destination node in a network. A population of $N$ users competes for these $K$ circuits. The $n^{\text {th }}$ user subjects the $K$ circuits to a "connection set-up request" process which we assume here to be Poisson with intensity $\lambda_{n}$ and arbitrary holding (or "service") time distribution with mean $1 / \mu_{n}$.

Thus, the system of users and network form a stationary multiclass $\mathrm{M} / \mathrm{GI} / \mathrm{K} / \mathrm{K}$ queue with total traffic intensity

$$
\rho \equiv \Lambda \frac{1}{\mu}
$$

where the aggregate arrival rate is

$$
\Lambda \equiv \sum_{n=1}^{N} \lambda_{n}
$$

and the mean holding time over all connections is

$$
\begin{aligned}
\frac{1}{\mu} & =\sum_{n=1}^{N} \frac{1}{\mu_{n}} \cdot \frac{\lambda_{n}}{\Lambda} \\
\Rightarrow \rho & =\sum_{n=1}^{N} \frac{\lambda_{n}}{\mu_{n}} .
\end{aligned}
$$

Indeed, we allow the service time distribution of each user to be different. The service time of a typical connection, even when the arrivals are not Poisson, is then a mixture of the different distributions using the weights $\lambda_{n} / \Lambda$.

The aggregate and per-user connection blocking probability in steady state is simply Erlang's formula [22],

$$
\mathcal{E}_{K}(\rho) \equiv \frac{\rho^{K} / K !}{\sum_{k=0}^{K} \rho^{k} / k !} .
$$


The $n e t$ arrival rate of the $n^{\text {th }}$ user is therefore

$$
\lambda_{n}\left(1-\mathcal{E}_{K}(\rho)\right) .
$$

So, by Little's formula, the mean number of occupied circuits for the $n^{\text {th }}$ user is

$$
\theta_{n}(\underline{\lambda}) \equiv \frac{1}{\mu_{n}} \lambda_{n}\left(1-\mathcal{E}_{K}(\rho(\underline{\lambda}))\right)
$$

where we have explicitly shown the dependence of $\theta_{n}$ and $\rho$ on all of the arrival rates $\underline{\lambda}$. Note that $\theta_{n}$ satisfies the assumptions of Section II only in the space: $A=$ $\left\{\underline{\lambda}_{N} \in\left(\mathbb{R}^{+}\right)^{N} \mid \min _{n} \partial_{n} \theta(\underline{\lambda}) / \partial \lambda_{n} \geq 0\right\}$. Suppose that $\prod_{n=1}^{N}\left[0, \lambda_{n}^{\max }\right] \subset A$ and note that $y_{n}=\left(U_{n}^{\prime}\right)^{-1}(M)$. Consider the following fixed-point iteration, using (1) with multiplicative increase and decrease (7):

$$
\begin{aligned}
\lambda_{n}^{j+1} & =\min \left\{\frac{y_{n}}{\theta_{n}} \lambda_{n}, \lambda_{n}^{\max }\right\} . \\
& =\min \left\{\frac{y_{n} \mu_{n}}{1-\mathcal{E}_{K}\left(\rho\left(\underline{\lambda}^{j}\right)\right)}, \lambda_{n}^{\max }\right\} .
\end{aligned}
$$

\section{A. Nash equilibria of the Erlang game model}

Suppose that $\underline{\lambda}^{*}$ is a fixed point for the unconstrained problem (10), i.e., $\lambda_{n}^{*}=\frac{y_{n} \mu_{n}}{1-\mathcal{E}\left(\rho\left(\lambda^{*}\right)\right)}$ for all $n$. To simplify notation, the superscript $*$ for a fixed point is dropped and $\rho$ and $\mathcal{E}$ will denote $\rho\left(\underline{\lambda}^{*}\right)$ and $\mathcal{E}_{K}\left(\rho\left(\underline{\lambda}^{*}\right)\right)$, respectively. Thus,

$$
\frac{\lambda_{n}}{\mu_{n}}=\frac{y_{n}}{1-\mathcal{E}} \text {. }
$$

The summation (11) over $n$ gives

$$
\rho=\frac{\sum_{n=1}^{N} y_{n}}{1-\mathcal{E}}=Y \frac{\sum_{k=0}^{K} \rho^{k} / k !}{\sum_{k=0}^{K-1} \rho^{k} / k !}
$$

where $Y=\sum_{n=1}^{N} y_{n}$. From (12), we get the following polynomial in $\rho$ :

$$
f(\rho) \equiv \sum_{k=1}^{K} \frac{(Y-k)}{k !} \rho^{k}+Y=0 .
$$

Hence, an interior fixed point of the constrained problem will be a root of (13). Note that (13) does not have a root if $Y \geq K$. If $Y<K$ then, for a sufficiently large $\rho$, the fact that $\frac{Y-K}{K !} \rho^{K}<0$ results in $f(\rho)<0$. The intermediate value theorem tells us that (13) should have at least one root between 0 and the sufficiently large $\rho .{ }^{1}$ Note that it is always possible to have unique root of (13) over $\prod_{n=1}^{N}\left[0, \lambda_{n}^{\max }\right]$ by an appropriate choice of $\lambda_{n}^{\max }$. Suppose that the real root of (13) exists in the interval of $\left(0, \sum_{n=1}^{N} \frac{\lambda_{n}^{\max }}{\mu_{n}}\right)$. Once the root of (13) is found, we can easily get $\lambda_{n}$ from (11). Therefore, the following theorem is proven.

Theorem 3: Suppose that $\sum_{n=1}^{N} y_{n}<K$. If (13) admits a positive real root $\rho^{*}$ in $\left(0, \sum_{n=1}^{N} \frac{\lambda_{n a x}^{\text {max }}}{\mu_{n}}\right)$, the fixed point of the unconstrained problem of (10) can be found from $\rho^{*}$ and (11).

\footnotetext{
${ }^{1}$ We evaluated several polynomials with large $\tilde{\rho}$ and various values of $K$ and found no more than a single root in the interval of interest $(0, \tilde{\rho})$.
}

Given the fixed point (NEP) of the unconstrained iteration is known, the issue of convergence to it is our next point of interest. We will study convergence of the Jacobi update scheme described in Section II-C.

Theorem 4: If there is a Nash equilibrium $\underline{\lambda} \in$ $\prod_{n=1}^{N}\left(0, \lambda_{n}^{\max }\right)$ and if $y_{n}>\frac{\lambda_{n}^{2} \mathcal{E}^{\prime}}{\mu_{n}} \sum_{l=1}^{N} \frac{1}{\mu_{l}}$ for all $n$ where $\mathcal{E}^{\prime}=\frac{\partial \mathcal{E}}{\partial \rho}$, then there is a neighborhood $B$ of $\underline{\lambda}$ such that the function $\underline{\lambda}(t)$ obeying the dynamics (10) will converge to $\underline{\lambda} \in B$ as $t \rightarrow \infty$ for any initial parameters $\lambda(0) \in B$.

Proof: Define the potential function

$$
L(\underline{\lambda})=\sum_{n=1}^{N} y_{n} \log \left(\lambda_{n}\right)-K \log (\rho)+\log (\mathcal{E}) .
$$

Since $\mathcal{E}^{\prime}=\partial \mathcal{E} / \partial \rho=(-1+K / \rho) \mathcal{E}+\mathcal{E}^{2}$, we get the following two equations:

$$
\begin{aligned}
\frac{\partial L}{\partial \lambda_{n}} & =\frac{y_{n}}{\lambda_{n}}-\frac{1-\mathcal{E}}{\mu_{n}} . \\
\frac{\partial L(\underline{\lambda}(t))}{\partial t} & =<\nabla L(\underline{\lambda}(t)), \underline{\dot{\lambda}}(t)> \\
& =\sum_{n=1}^{N} \frac{(1-\mathcal{E})}{\mu_{n} \lambda_{n}}\left(\frac{y_{n} \mu_{n}}{1-\mathcal{E}}-\lambda_{n}\right)^{2} \\
& \geq 0
\end{aligned}
$$

Note that $\nabla L=0$ and the equality in (15) holds for only the fixed point $\lambda=F(\lambda)$. Finally, the components of the Hessian $\mathrm{H}$ of $L$ are

$$
H_{n, l} \equiv \frac{\partial^{2} L}{\partial \lambda_{n} \lambda_{l}}= \begin{cases}\frac{\mathcal{E}^{\prime}}{\mu_{n} \mu_{l}} & \text { if } n \neq l \\ -\frac{y_{n}}{\lambda_{n}^{2}}+\frac{\mathcal{E}^{\prime}}{\mu_{n}^{2}} & \text { if } n=l .\end{cases}
$$

The condition,

$$
y_{n}>\frac{\lambda_{n}^{2} \mathcal{E}^{\prime}}{\mu_{n}} \sum_{l=1}^{N} \frac{1}{\mu_{l}} \text { for all } n
$$

makes the Hessian diagonally dominant and, therefore [7], the Hessian is negative definite at the Nash equilibrium. By the continuity property of the Hessian, there is a neighborhood $B$ around $\underline{\lambda}$ where the Hessian is negative definite everywhere on $B$. These facts imply that the Nash equilibria are local minima using $L$ as a local Lyapunov function.

\section{B. Numerical studies}

Consider a simple example of two user game with $K=1$, i.e., the two users share a single channel. Let $\left(\mu_{1}, \mu_{2}\right)=(1,2)$, $\left(y_{1}, y_{2}\right)=\left(\frac{1}{3}, \frac{1}{2}\right)$ and $\left(\lambda_{1}^{\max }, \lambda_{2}^{\max }\right)=(10,20)$. Using Matlab, we have checked the condition that $\left[0, \lambda_{1}^{\max }\right] \times\left[0, \lambda_{2}^{\max }\right] \subset A$. We got $\rho^{*}=5$ and $\left(\lambda_{1}^{*}, \lambda_{2}^{*}\right)=(2,6)$ from (11) and (13) and used $\epsilon=0.1$ for the Jacobi iteration. From Figures 1 and 2, we see that the quantities $\left(\lambda_{1}^{*}, \lambda_{2}^{*}\right)$ which were solved analytically 
coincide with the values obtained by the fixed point iteration. Note that $\theta_{n}^{*}=y_{n}^{*}$ for $n=1,2$.

For a variety of two-user experiments under various initial points, we observed that this iteration always converged to a single fixed point even when diagonal dominance of the Hessian was not satisfied. We point out that the condition of diagonal dominance is only sufficient to guarantee (local) convergence.
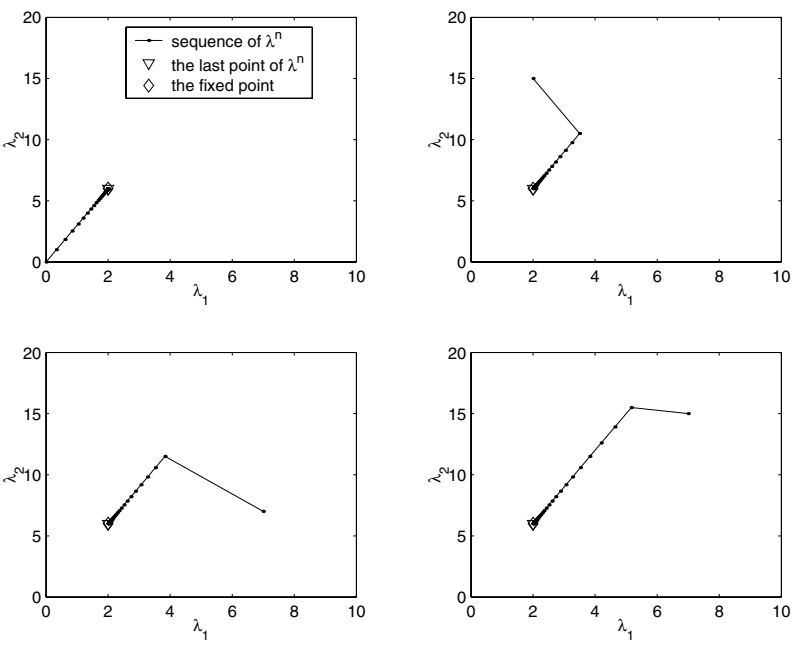

Fig. 1. The convergence of (10) when $\rho<K$
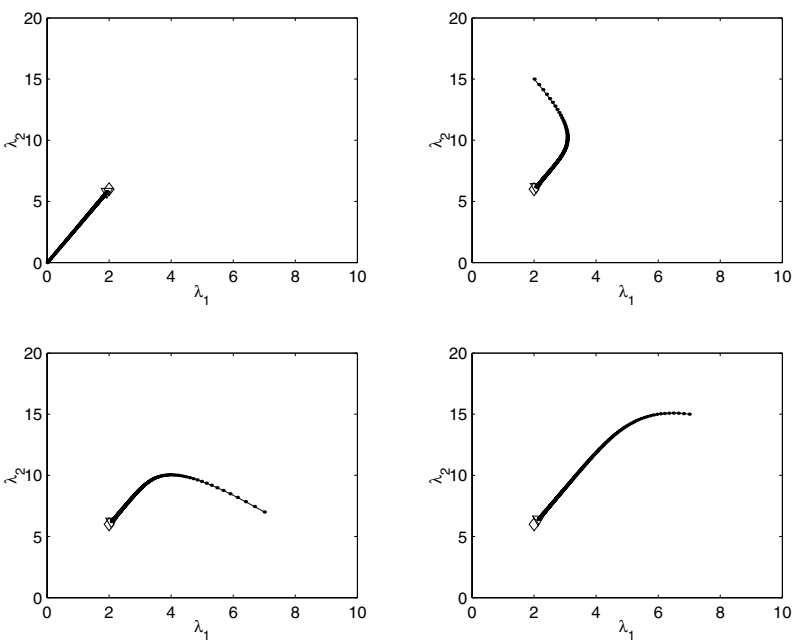

Fig. 2. The convergence of the Jacobi iteration of (10) when $\rho<K$

Recall that if $K \geq \rho$ and the $\lambda_{n}^{\max }$ are sufficiently large so that $\underline{\lambda}^{*}$ is in interior of $\prod_{n=1}^{N}\left[0, \lambda_{n}^{\max }\right]$, then all user demands are met, i.e., $\theta_{n}=y_{n}$ for all $n$. Clearly, there are two "causes" of boundary NEPs: the first is that the network is not congested but that $\lambda_{n}^{*}>\lambda_{n}^{\max }$ for at least one user $n$ where $\underline{\lambda}^{*}$ is the "unconstrained" NEP, and the other cause is congestion wherein demand exceeds capacity, $Y=\sum_{n=1}^{N} y_{n} \geq K$, and the network cannot therefore meet every user's demand. Again, recall that at NEPs $\underline{\lambda}^{*}$ on the boundary of $\prod_{n=1}^{N}\left[0, \lambda_{n}^{\max }\right]$, certain users receive less than their demand but no users receive more than their demand (Lemma 1) which is, again, a very basic notion of fairness.

Figures 3 and 4 illustrate user behavior when $Y>K$; more specifically, $\left(y_{1}, y_{2}\right)=(1 / 3,5 / 7)$ and $\epsilon=0.1$ for Jacobi iteration are used. The plots show that the fixed points occur on the boundary of the space: $\left(\lambda_{1}^{*}, \lambda_{2}^{*}\right)=(5.5,20)$ is obtained from the iteration resulting in $\left(\theta_{1}, \theta_{2}\right)=(0.3333,0.6061)$. Note that $\theta_{2}<y_{2}$ and $\theta_{1}=y_{1}<y_{2}$.
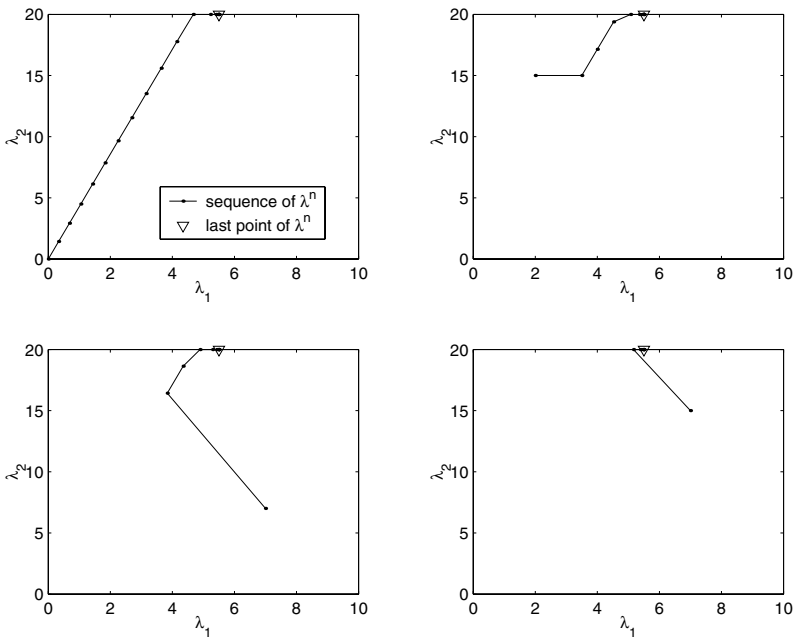

Fig. 3. The convergence of (10) when $\rho>K$
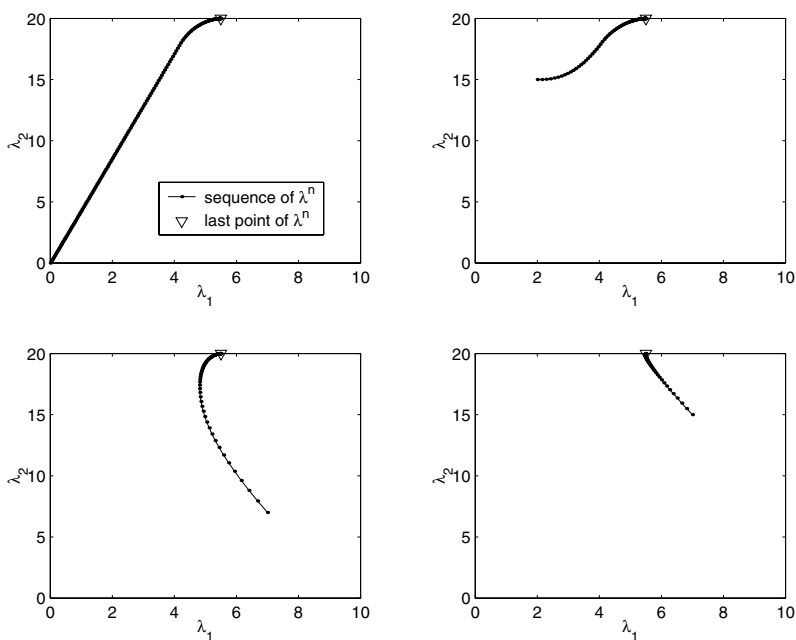

Fig. 4. The convergence of the Jacobi iteration of (10) when $\rho>K$

We conducted simulations using additive increase and multiplicative decrease function (6) with decrease factor $1-\alpha=0.5$ and increase parameter $\delta=1$. The results for the congested $(Y \geq K)$ example above are shown in Figures 5, 6, and 7 . All simulations have used $\underline{y}=(10,15,20,25,30)$ and $\underline{\mu}=$ $(15,20,25,30,35)$. We found a single equilibrium point (irrespective of initial values) for the iteration using MIMD and a slightly different single equilibrium point (again, independent 
of initial values) under AIMD. In the depicted simulation results, the initial value of $(10,30,10,30,10)$ was used. The dynamics of the fixed point and the fairness properties were investigated for various choices of $\lambda^{\max }$. As fairness indices, the absolute fairness (throughput $\theta$ ) and demand proportional fairness (i.e., the ratio of $\theta$ to $y, \theta / y$ ) were used.
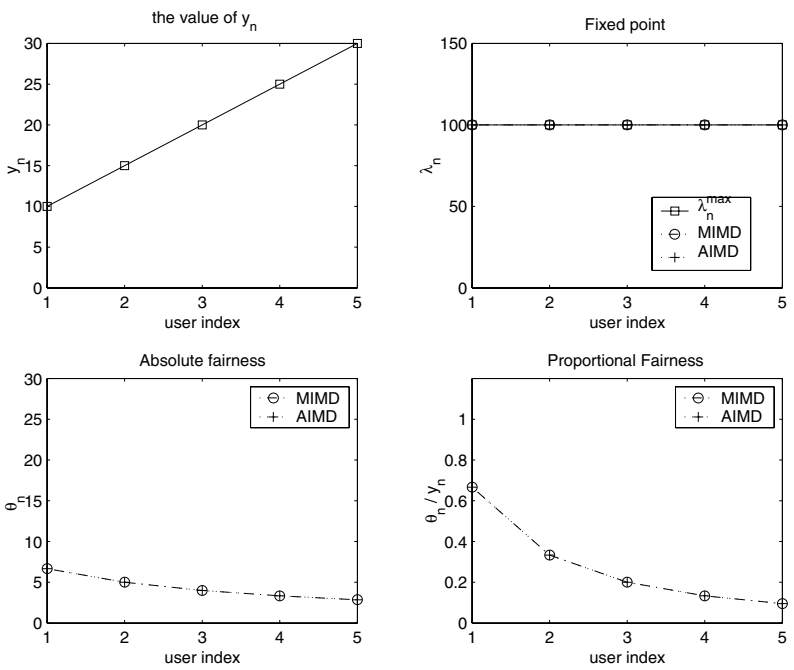

Fig. 5. Fairness of MIMD and AIMD: Case I

The values of $\lambda_{n}^{\max }=100$ for all $n$ were used for Figure 5. The fixed point reached was $\lambda_{n}^{*}=\lambda_{n}^{\max } \equiv 100$ for all $n$ for both MIMD and AIMD.
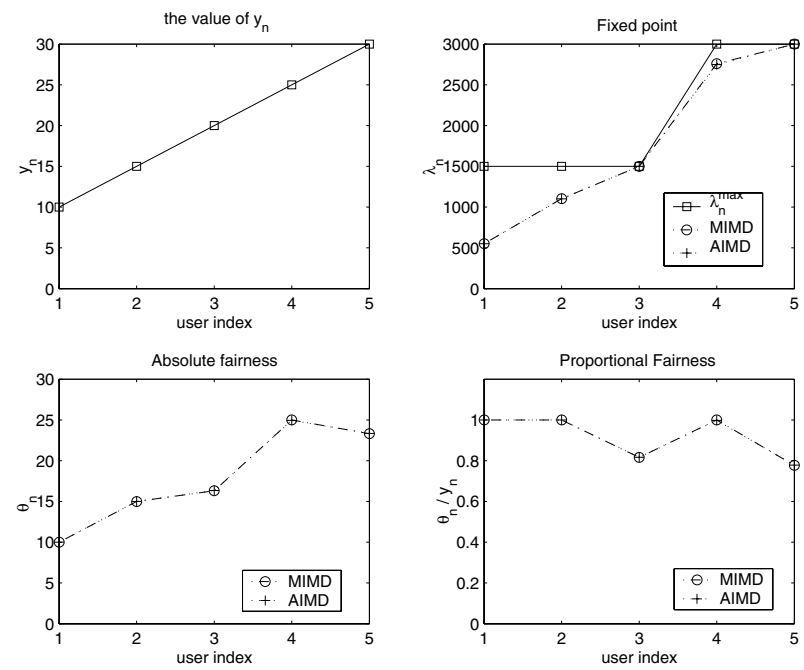

Fig. 6. Fairness of MIMD and AIMD: Case II

For Figures 6 and 7, (1500, 1500, 3000, 3000, 3000) and $(1500,1500,3000,5000,8000)$ were respectively used for $\underline{\lambda}^{\max }$. In Figure $6, \lambda_{n}^{*}<\lambda_{n}^{\max }$ for all $n$ except $n=3$. Note that the fixed point is also on the boundary in Figure 7 even if, for all $n$, larger values of $\lambda_{n}^{\max }$ are used (here $\lambda_{n}^{*}=\lambda_{n}^{\max }$ for $n=2,3,4)$. This is expected because the condition $\rho>K$ implies no analytical solution of the unconstrained problem
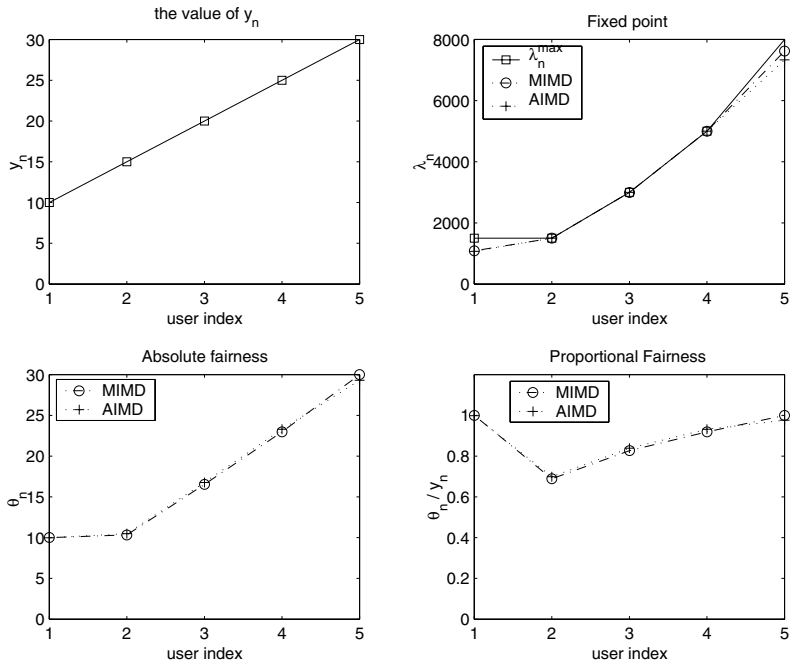

Fig. 7. Fairness of MIMD and AIMD: Case III

exists. All the figures show that the fairness of MIMD is very close (almost equal) to the fairness of AIMD in both absolute and demand proportional fairness (which is not the case for TCP congestion control in the present-day Internet [6]). In addition, we observed that the convergence rate of AIMD is much slower than MIMD as $\lambda_{n}^{\max }$ increases. We conclude that values of $\lambda_{n}^{\max }$ significantly affect the fixed point and fairness from these plots.

\section{AN ALTERNATIVE NONCOOPERATIVE CIRCUIT-SWITCHED GAME}

In the context of the example of Section III, assume now that the $n^{\text {th }}$ user will subject the network to highest connection arrival rate $\lambda_{n}$ subject to a desired upper bound on his/her connection blocking rate $y_{n}$. This user could, for example, be a call center that accepts a call only if it can guarantee set-up through the network for the call with high probability $\left(1-y_{n}\right)$ [12].

The actual blocking rate of the $n^{\text {th }}$ user is

$$
\theta_{n}(\underline{\lambda}) \equiv \lambda_{n} \mathcal{E}_{K}(\rho(\underline{\lambda})) .
$$

Again, under the fixed point iteration (1) with multiplicative increase and decrease (7), after the $j^{\text {th }}$ step the $n^{\text {th }}$ user has control parameter:

$$
\begin{aligned}
\lambda_{n}^{j+1} & =\min \left\{\frac{y_{n}}{\theta_{n}(\underline{\lambda})} \lambda_{n}, \lambda_{n}^{\max }\right\} \\
& =\min \left\{\frac{y_{n}}{\mathcal{E}_{K}(\rho(\underline{\lambda}))}, \lambda_{n}^{\max }\right\}
\end{aligned}
$$

\section{A. The fixed point of the problem}

Suppose that $\underline{\lambda}^{*}$ is a fixed point for the unconstrained problem of (16), i.e., $\lambda_{n}^{*}=\frac{y_{n}}{\mathcal{E}\left(\rho\left(\lambda^{*}\right)\right)}$. To simplify notation, the superscript $*$ for a fixed point is dropped and $\rho$ and $\mathcal{E}$ denote $\rho\left(\underline{\lambda}^{*}\right)$ and $\mathcal{E}_{K}\left(\rho\left(\underline{\lambda}^{*}\right)\right)$, respectively as in Section III. 
Then,

$$
\frac{\lambda_{n}}{\mu_{n}}=\frac{y_{n}}{\mu_{n} \mathcal{E}} .
$$

The summation (17) over $n$ gives

$$
\rho \mathcal{E}=\sum_{n=1}^{N} \frac{y_{n}}{\mu_{n}}
$$

where $y=\sum_{n=1}^{N} y_{n}$. From (18), we get the following polynomial in $\rho$ :

$$
g(\rho) \equiv \frac{\rho^{K+1}}{K !}-\sum_{n=1}^{N} \frac{y_{n}}{\mu_{n}} \sum_{k=0}^{K} \frac{\rho^{k}}{k !}=0 .
$$

Since $g(0)<0$ and $g(\rho)>0$ for sufficiently large $\rho$, we can always find a root of $g(\rho)$. Note that $n^{\text {th }}$ component of fixed point, $\lambda_{n}$ can be obtained using (17). We have the following theorem as in the previous section.

Theorem 5: If (19) admits a positive real root $\rho$ in $\left(0, \sum_{n=1}^{N} \lambda_{n}^{\max } / \mu_{n}\right)$, the fixed point of the unconstrained problem of (16) can be found from $\rho$ and (17).

The following function tells us useful information about the location of fixed points, especially if multiple NEPs exist.

$$
\begin{aligned}
\Lambda(\underline{\lambda}) & =-\frac{\left(\sum_{n=1}^{N} \lambda_{n}\right)\left(\sum_{n=1}^{N} y_{n} \lambda_{n}\right)}{\sum_{n=1}^{N} y_{n}} \\
& +\frac{1}{2}\left(\sum_{n=1}^{N} \lambda_{n}^{2}\right)+\frac{1}{2} \frac{\left(\sum_{n=1}^{N} \lambda_{n}\right)^{2}\left(\sum_{n=1}^{N} y_{n}^{2}\right)}{\left(\sum_{n=1}^{N} y_{n}\right)^{2}}
\end{aligned}
$$

Theorem 6: $\nabla \Lambda\left(\underline{\lambda}^{*}\right)=0$ for all "unconstrained" fixed points $\underline{\lambda}^{*}$ of (16), i.e., all $\underline{\lambda}^{*}$ such that $\lambda_{n}^{*}=F_{n}\left(\underline{\lambda}^{*}\right)=$ $\frac{y_{n}}{\mathcal{E}_{K}\left(\rho\left(\underline{\lambda}^{*}\right)\right)}$ for all $n$.

Proof: The lemma follows direct differentiation and from the following relation that holds at unconstrained fixed points $\underline{\lambda}^{*}$ of (16):

$$
\begin{aligned}
y_{n} & =\mathcal{E}_{K}\left(\rho\left(\underline{\lambda}^{*}\right)\right) \lambda_{n}^{*} \\
\sum_{n=1}^{N} y_{n} & =\mathcal{E}_{K}\left(\rho\left(\underline{\lambda}^{*}\right)\right) \sum_{n=1}^{N} \lambda_{n}^{*}
\end{aligned}
$$

Note that the equation $\nabla \Lambda\left(\underline{\lambda}^{*}\right)$ is linear in $\underline{\lambda}$, i.e.,

$$
\nabla \Lambda\left(\underline{\lambda}^{*}\right)=A \underline{\lambda}^{*}
$$

where the $N \times N$ matrix $A$ has the following components:

$$
A_{k, n}= \begin{cases}-\frac{y_{k}+y_{n}}{\sum_{i=1}^{N} y_{i}}+\frac{\sum_{i=1}^{N} y_{i}^{2}}{\left(\sum_{i=1}^{N} y_{i}\right)^{2}} & \text { for } k \neq n \\ -\frac{2 y_{k}}{\sum_{i=1}^{N} y_{i}}+1+\frac{\sum_{i=1}^{N} y_{i}^{2}}{\left(\sum_{i=1}^{N} y_{i}\right)^{2}} & \text { for } k=n\end{cases}
$$

Therefore, all unconstrained fixed points lie in the null space of $A$. Also note that $\nabla \Lambda(y)=0$ and $\nabla \Lambda(0)=0$. Therefore, if $\operatorname{rank}(A)=N-1$, then all unconstrained fixed points lie on the line connecting the origin (0) and $y$.

The following theorem is the main convergence result of this section.

Theorem 7: The following function is a potential function of (16):

$L(\underline{\lambda})=-\sum \frac{\lambda_{n}}{\mu_{n}}+K \log (\rho)+\sum_{n=1}^{N} \frac{y_{n}}{\mu_{n}} \log \left(\lambda_{n}\right)-\log (\mathcal{E})$.

Proof: Note that the following two equations.

$$
\begin{aligned}
\frac{\partial L}{\partial \lambda_{n}}= & -\frac{1}{\mu_{n}}+K \frac{1}{\rho \mu_{n}}+\frac{y_{n}}{\mu_{n} \lambda_{n}}-\frac{\mathcal{E}^{\prime}}{\mathcal{E} \mu_{n}} \\
= & \frac{1}{\mu_{n}}\left\{\frac{y_{n}}{\lambda_{n}}-\mathcal{E}\right\} . \\
\frac{\partial L(\underline{\lambda}(t))}{\partial t} & =<\nabla L(\underline{\lambda}(t)), \underline{\dot{\lambda}}(t)> \\
& =\sum_{n=1}^{N} \frac{\mathcal{E}}{\mu_{n} \lambda_{n}}\left(\frac{y_{n}}{\mathcal{E}}-\lambda_{n}\right)^{2} \geq 0 .
\end{aligned}
$$

Moreover, the components of the Hessian $\mathrm{H}$ of $L$ are

$$
\frac{\partial^{2} L}{\partial \lambda_{n} \lambda_{k}}= \begin{cases}\frac{-\mathcal{E}^{\prime}}{\mu_{n} \mu_{k}} & \text { if } n \neq k \\ -\frac{y_{n}}{\mu_{n} \lambda_{n}^{2}}-\frac{\mathcal{E}^{\prime}}{\mu_{n} \lambda_{n}} & \text { if } n=k .\end{cases}
$$

Clearly, the Hessian $H$ is negative definite.

\section{B. Numerical studies}

Consider the case $K=1,\left(y_{1}, y_{2}\right)=(1 / 3,1 / 2)$ and $\left(\mu_{1}, \mu_{2}\right)=(1,2)$. Then $\rho=1.1092$ and $\left(\lambda_{1}, \lambda_{2}\right)=$ $(0.6338,0.9508)$ from (19). Note that $\frac{\lambda_{1}}{\lambda_{2}}=\frac{y_{1}}{y_{2}}$, hence $\left(\lambda_{1}, \lambda_{2}\right)$ is on the line which connects the origin and $\underline{y}$.
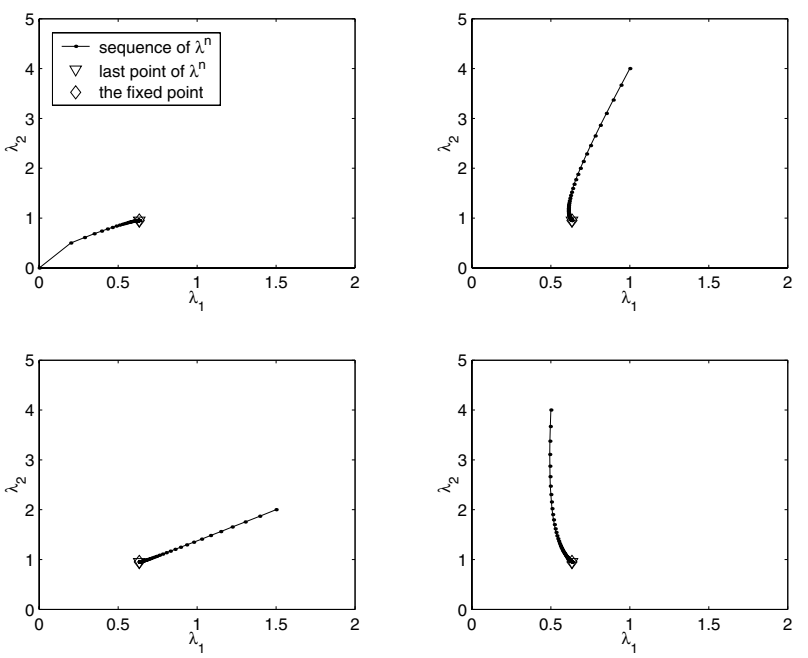

Fig. 8. The convergence to the fixed point using (1) 

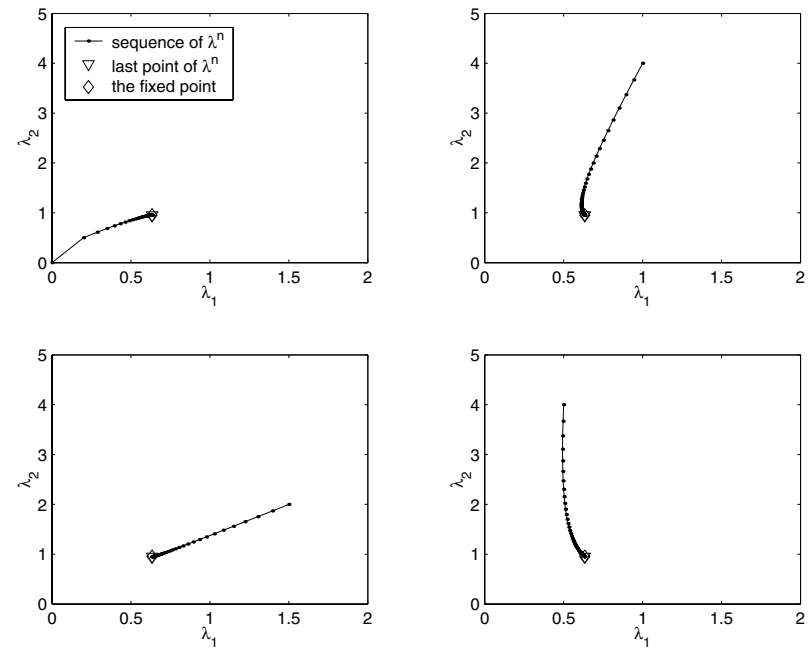

Fig. 9. The convergence to the fixed point using the Jacobi iteration

\section{SUMmARY}

We have proposed a generic user-network interface for the emerging Internet offering premium CoSs which are billed based on usage. Corresponding convergence dynamics to a Nash equilibrium (fixed point) were studied. This paper has focused on the fixed points of two problems in which users modify their access parameters to satisfy their demands for QoS in an MPLS Internet offering LSPs having a resizable (renegotiable) provision of bandwidth. In both cases, the fixed points of the noncooperative game can be determined from the roots of certain specified polynomials. Convergence to these fixed points was studied analytically using potential functions and by simulation. Fairness issues were also explored by considering the boundary fixed points when total user demand exceeds network capacity, i.e., the network is in a congested state.

\section{REFERENCES}

[1] T. Basar and G. J. Olsder Dynamic noncooperative game theory, 2nd Ed. Academic Press, 1995.
[2] S. Blake, D. Black, M. Carlson, E. Davies, and Z. Wang. An architecture for Differentiated Services. IETF RFC2475, Dec. 1998, available from URL www.ietf.org/html.charters/diffserv-charter.html.

[3] K.C. Border. Fixed Point Theorems with Applications to Economics and Game Theory. Cambridge University Press, London, 1985.

[4] P. Brittain and A. Farrel. MPLS traffic engineering: A choice of signaling protocols. Technical report, Data Connection Ltd, available from http://www.dataconnection.com /mpls/wpd12.htm, 2000.

[5] R. Callon, P. Doolan, N. Feldman, A. Fredette, G. Swallow, and A. Viswanathan. A framework for multiprotocol label switching. Technical report, Internet Draft, http://www.ietf.org, July 1999.

[6] D. Chiu and R. Jain. Analysis of the increase and decrease algorithms for congestion avoidance in computer networks. Computer Networks and ISDN Systems, Vol. 17, No. 1:314-329, 1989.

[7] R.A. Horn and C.R. Johnson. Matrix Analysis. Cambridge, New York, 1988.

[8] Y. Jin and G. Kesidis. Equilibria of a noncooperative game for heterogeneous users of an ALOHA network. IEEE Comm. Letters, Vol.6, No. 7:pp. 282-284, 2002. 2002.

[9] Y. Jin and G. Kesidis. A pricing strategy for an ALOHA network of heterogeneous users with inelastic bandwidth requirements. In Proc. CISS, Princeton, March 2002.

[10] F.P. Kelly. Charging and rate control for elastic traffic. European Transactions on Telecommunications, 8:pp. 33-37, 1997.

[11] F.P Kelly, A.K. Maulloo, and D.K.H. Tan. Rate control for communication networks: shadow prices, proportional fairness and stability. Journal of the Operational Research Society, 49:pp. 237-252, 1998.

[12] G. Kesidis, K. Chakraborty, and L. Tassiulas. Traffic shaping for a loss system. IEEE Communication Letters, Vol. 4, No. 12:pp. 417-419, Dec. 2000.

[13] G. Kesidis and Y. Jin. Feasible pricing of differentiated services for the emerging Internet. In Proc. 40th Allerton Conference on Communications, Control and Computing, Oct. 2002.

[14] J.F. Kurose and K.W. Ross. Computer Networking. Addison Wesley, 2001.

[15] R. La and V. Anantharam. Charge sensitive TCP and rate control in the internet. In Proc. IEEE INFOCOM, 2000.

[16] S.H. Low and B.E. Lapsely Optimization flow control. I. Basic algorithm and convergence. IEEE/AC Trans. Networking, Vol. 7, No. 6, 199.

[17] P. Marbach. Pricing differentiated services networks: bursty traffic. In Proc. IEEE INFOCOM, 2001.

[18] P. Marbach. Priority service and max-min fairness. In Proc. IEEE INFOCOM, 2002

[19] K. Nichols, S. Blake, F. Baker, and D. Black. Definition of the Differentiated Services field (DS field) in the IPv4 and IPv6 headers. IETF RFC2474, Dec. 1998, available from URL www.ietf.org/html.charters/diffserv-charter.html.

[20] D.R. Smart. Fixed Point Theorems. Cambridge University Press, London, 1974.

[21] X. Wang and H. Schulzrinne. Pricing network resources for adaptive applications ni a differentiated services network. In Proc. IEEE INFOCOM, 2001.

[22] R.W. Wolff. Stochastic Modeling and the Theory of Queues. PrenticeHall, Englewood Cliffs, NJ, 1989. 